\title{
Validação das competências do enfermeiro nos cuidados com portadores de marca-passo
}

RESUMO | Objetivo: validar competências de enfermeiros atuantes nos cuidados com portadores de marca-passo. Método: tratase de estudo de validação de conteúdo, realizado entre março e novembro de 2017, pela técnica Delphi, sob protocolo CAAE $n^{\circ}$ 63940217.3.0000.5462. A apreciação das competências foi realizada por sete juízes enfermeiros, doutores e experts em cardiologia, que consideraram o índice para validação de conteúdo (IVC) superior a 70\%. Resultados: foram validados 119 itens de cuidado do total de 133, distribuídos em nove competências, com média geral de cada competência (AVE) e de concordância dos juízes (UA) com IVC>0,7. Dos 14 itens não validados após segunda rodada de avaliação, foram validados com AVE geral de 0,9 e UA de 1. Observouse relevância da competência sobre os conhecimentos de enfermagem para promoção da assistência segura aos familiares e à ação educativa ao paciente. Conclusão: As competencias foram validadas quanto à relevância e confiabilidade pelos juízes.

Palavras-chaves: Estudos de Validação; Competência Profissional; Marca-passo Artificial.

ABSTRACT | Aim: To validate nursing core competencies in the care management of patients with pacemakers. Methods: We conducted a single-center content validation study from March through November, 2017, using the Delphi e approval CAAE 63940217.3.0000.5462. Competency assessment was performed by seven judges (nurses with PhD degree and specialty in cardiology), who considered the content validation index (CVI) greater than 70\%. Results: 119 care items out of the total number of 133 were distributed in nine competencies, with an average of each competence (AVE) and concordance of the judges (UA) with CVl>0.7. 14 items which had not been validated after the second round of evaluation were validated with an $A V E=0.9$ and $A U=1$. Competence relevance on nursing knowledge was identified for the support in safe care by the patient's relatives and the education training for the patient. Conclusion: The competencies were validated as to relevance and reliability by the judges.

Keywords: Validation Studies; Professional Competence; Pacemaker Artificial.

RESUMEN | Objetivo: validar las competencias de las enfermeras que trabajan en el cuidado de pacientes con marcapasos. Método: estudio de validación de contenido, realizado entre marzo y noviembre de 2017, mediante la técnica Delphi, aprobación el protocolo CAAE 63940217.3.0000.5462. La evaluación de la competencia fue realizada por siete enfermeras, doctor y expertos en cardiología, quienes consideraron que el índice del validación de contenido (IVC) era superior al 70\%. Resultados: se distribuyeron 119 ítems de 133, distribuidos en nueve competencias, con un promedio general de cada competencia (AVE) y concordancia de los jueces (AU) con IVC> 0.7. De los 14 ítems no validados después de la segunda ronda, se validaron con un golpe general de 0,9 y una UA de 1 . Se observó relevancia para promover la atención y la acción segura de la familia al paciente. Conclusión: las competencias fueron validadas en cuanto a la relevancia y confiabilidad de los jueces.

Descriptores: Estudios de Validación; Competencia Profesional; Marcapaso Artificial.

\section{Tamires de Castro Vieira}

Enfermeira. Especialista pelo Programa de Residência em Enfermagem Cardiovascular do Instituto Dante Pazzanese de Cardiologia. São Paulo (SP), Brasil.

\section{Sérgio Henrique Simonetti}

Enfermeiro. Chefia de Enfermagem de Unidade de Exames Cardiológicos não Invasivos. Pós-Doutor pelo Instituto Dante Pazzanese de Cardiologia-USP. Doutor e Mestre pela EEUSP. São Paulo (SP), Brasil.

\section{Rika Miyahara Kobayashi}

Enfermeira. Diretora do Serviço de Educação Continuada e Coordenadora da Comissão de Residência Multiprofissional do Instituto Dante Pazzanese de Cardiologia. Doutor e Mestre em Enfermagem pela EEUSP. São Paulo (SP), Brasil

Recebido em: 04/07/2018

Aprovado em: 04/07/2019

INTRODUÇÃO

s doenças cardiovasculares
são responsáveis por 17 mi-
lhões de mortes por ano e um dos maiores problemas de saúde pública no mundo, destacando se que quase $80 \%$ de mortes por doenças cardiovasculares ocorrem em países de média e baixa renda, e cujas causas são evitáveis por meio de intervenções. ${ }^{1}$

Em 2016 estimou-se o aumento do número anual de mortes devido às doenças cardiovasculares de 17,5 milhões em 2012, para 22,2 milhões até 2030. Também neste estudo, salientou-se que as causas poderiam ser evitadas por meio de intervenções no estilo de vida e tratamentos adequados. ${ }^{2}$

Os marca-passos convencionais são importantes formas de tratamento de doenças cardíacas, essencialmente em casos de bloqueio de condução elétrica devido à lesão no coração, em doença do nó sinusal, bloqueios atrial ou intraventricular, hipersensibilidade do seio carotídeo, na fibrilação atrial, cardiomiopatia hipertrófica obstrutiva e síncope neurocardiogênico ${ }^{3,4}$, bem como a colocação de dispositivos de estimulação cardíaca é indicada em insuficiência cardíaca. ${ }^{5}$

Entende-se que há alternativas de tratamento como utilização de medicamentos antiarrítmicos, ressecção cirúrgica, ablação endocárdica por cateter e uso de dispositivo cardíaco eletrônico implantável $(\mathrm{DCEI})^{3}$ mas ao verificar se que em 2013, conforme a descrição do estudo da Sociedade Europeia de Cardiologia, em 3580 centros houve a implantação de 500.411 marca-passos, confirma se a necessidade de manejo e conhecimento dessa modalidade de tratamento. ${ }^{6}$ 
Deste modo, por se tratar de um cuidado complexo e específico, o delineamento e clareza das competências profissionais tornam-se essenciais no cuidado ao paciente portador de marca-passo. Entende-se por competência profissional, a capacidade do indivíduo que, ao inserir-se no contexto do trabalho, envolve suas capacidades para atendimento às demandas da organização, contribuindo para a construção das mesmas. ${ }^{7}$

A literatura sobre competências profissionais em marca-passo ainda é escassa e descreve-se que o profissional deve conhecer o funcionamento do equipamento, saber identificar os traçados eletrocardiográficos, o processo fisiopatológico da doença que acomete o paciente, além do manejo no cuidado para que realize com segurança os diagnósticos de enfermagem prioritários, requerendo do enfermeiro, um conhecimento técnico científico mais aprofundado. ${ }^{8}$

Diante disso, foi realizada uma revisão integrativa sobre as competências profissionais em marca-passo, descrevendo na continuidade do estudo seria necessária a validação destas competências construídas. ${ }^{9}$

Assim, para buscar uma aproximação ao objetivo do pretendido estudo, questionouse: "Qual o perfil de competências do enfermeiro no cuidado com o portador de marcapasso?". Desse modo, objetivou-se validar das competências do enfermeiro no cuidado com portadores de marca-passo elaboradas com o intuito de contribuir na prática assistencial ao portador de marca-passo hospitalizado, para ações educativas ao usuário e seus familiares, bem como no ensino de enfermagem, principalmente especializada na atenção ao cardiopata.

\section{MÉTODO}

Trata-se de um estudo de validação de conteúdo de competências profissionais no cuidado ao portador de marca-passo por meio da técnica Delphi, realizado no período de março a novembro de 2017. Constituiu-se a população por juízes experts cujos critérios de inclusão foram ser profissionais graduados em enfermagem, doutores com especialização em cardiologia e/ou experiência na área específica. Adotou-se o procedimento de contato via e-mail e envio de uma carta convite com descrição do projeto de validação das competências profissionais.

O estudo foi submetido e aprovado pelo Comitê de Ética em Pesquisa de um hospital público de referência em cardiologia do município de São Paulo, respeitando as diretrizes da Resolução n. ${ }^{\circ}$ 466/2012, sendo aprovado sob CAAE n. ${ }^{\circ}$ 63940217.3.0000.5462.

Foram convidados 12 especialistas e 7 se disponibilizaram a participar da pesquisa. Foi entregue o Termo de Consentimento Livre e Esclarecido, o instrumento de coleta de dados aos experts, e estabeleu-se o prazo de 15 dias para a devolução do material analisado e com o parecer final.

Neste estudo foi adotado o rol de competências profissionais elaborado por Kobayashi RM, Simonetti SH e Pascon DM, construído a partir de uma Revisão Integrativa em que os autores9 elencaram nove competências profissionais do enfermeiro no cuidado ao portador de marca-passo com um total de 133 ítens de cuidado a saber: Competências profissionais educacionais relacionadas à equipe de enfermagem (03); Competências profissionais relacionadas a conhecimentos de enfermagem para promoção da assistência segura (08); Competências profissionais relacionadas à avaliação clínica do paciente no pré-operatório (14); Competências profissionais relacionadas à avaliação cirúrgica do paciente e de gestão do cuidado perioperatório (29); Competências profissionais relacionadas à assistência no pós-operatório (20); Competências profissionais para organização diária da assistência de enfermagem (22); Competências profissionais relacionadas à identificação, intervenção frente às principais complicações (13); Competências profissionais relacionadas à ação educativa ao paciente (32); Competências profissionais educacionais relacionadas aos familiares (12). ${ }^{9}$ Em cada competência profissional elencada, os autores descreveram conhecimentos, habilidades e/ ou atitudes necessárias respaldadas nas literaturas.

Este rol de competências profissionais foi enviado para análise e julgamento pelos experts e seria considerada validada se o Índice de Validade de Conteúdo (IVC) fosse superior a 0,7. O critério de concordância foi avaliado pela utilização da escala Likert de 0 a 3 que variou entre concordo plenamente, concordo, discordo e discordo plenamente. Para os critérios onde não houve concordância, utilizou-se a técnica Delphi tendo sido necessárias duas rodadas na avaliação de concocrdância com os conteúdos.

Adotou-se o IVC para verificação da validação do questionário pelos juízes em relação à permanência ou não dos itens dos instrumentos. Para se avaliar todo instrumento neste estudo, aplicou-se a média dos valores dos itens calculados separadamente (IVC média). Realizou-se a soma de todos os IVCs calculados separadamente e divididos pelo número de itens do instrumento (IVC UA).10 O nível de concordância estabelecido (IVC $>0,7$ ) baseou-se em outro estudo que considerou este IVC para uma

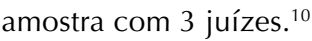

Para a tabulação, utilizou-se o programa Microsoft Excel 2010 e realizou-se análise utilizando o Índice de Validação de Conteúdos. Ao fim da primeira rodada de avaliação, reformulou se alguns itens de competnecia de acordo com as sugestões dos juízes, e após a segunda rodada de avaliação obteve se o IVC acima de 0,7.

\section{RESULTADOS}

Caracterizando a população dos sete juízes para validação de conteúdo, $6(86 \%)$ eram do sexo feminino; $100 \%$ 
com doutorado e com experiência em cardiologia e no cuidado com paciente portador de marcapasso, 4 (57\%) com docência em Enfermagem, atendendo aos critérios de inclusão para a validação das competências.

Em relação à validação de competências profissionais, foram apresentas na tabela 1 o julgamento cada competência profissional do enfermeiro nos cuidados com portador de marca-passo e na tabela 2 os ítens de cuidado com as alterações após sugestões dos juízes e os respectivos IVCs.

Observa-se neste estudo o grau de relevância das competências por meio das avaliações dos juízes na seguinte ordem: competências relacionadas a conhecimentos de enfermagem para promoção da assistência segura $(0,95)$, competências relacionadas aos familiares $(0,93)$ e competências relacionadas à ação educativa ao paciente $(0,93)$.

Do total de 133 itens de ccuidados avaliados, verificou-se que 14 não foram validados na primeira rodada (abaixo de $0,7)$, e após sugestões e alterações destes itens, realizou-se a segunda e última rodada, com validação de todos os itens com AVE geral de 0,90 e UA de 1.

Com base no julgamento dos juízes para validação da lista de competências, apontaram-se sugestões para melhora nos seguintes itens, considerando antes e após as alterações com os respectivos IVCs que segue na Tabela 2.

Tabela 1. Julgamento dos juizes sobre os itens das competências relacionadas ao cuidado com portador de marca-passo, com a

média dos índices de validação de conteúdo por competências (AVE) e concordância dos juízes (UA) na primeira e segunda rodada. São Paulo, SP, Brasil, 2017.

\section{Competências Profissionais}

\section{Avaliação Inicial \\ Avaliação Final}

AVE

0,76

0,80

0,87

0,92

0,90

1

3. Avaliação clínica do paciente no pré-operatório (14)

4. Avaliação cirúrgica do paciente e de gestão do cuidado Perioperatório (29)

0,85

0,89

0,89

1

5. Assistência no pós-operatório (20)

0,82

0,85

0,89

1

6. Organização diária da assistência de enfermagem (22)

0,82

0,86

0,90

0,84

0,88

7. Identificação e intervenção frente às complicações (13)

8. Ação educativa ao paciente (32)

0,92

0,82

0,76

0,96

0,93

0,93
9. Assistência aos familiares (12)

Fonte: Informações Coletadas a partir do Formulário elaborado para pesquisa. 2017. 
Tabela 2. Itens com as alterações após sugestões dos juízes e os respectivos IVCs. São

Paulo, SP, Brasil, 2017.

$\begin{array}{llll}\text { Item Item Inicial } \quad \text { ICV Inicial } & \text { Item Final }\end{array}$

2. Conhecimentos de enfermagem para promoção da assistência segura (8)

8 Conhecer os cuidados perioperatórios.
Conhecer os cuidados de enfermagem aos pacientesque serão submetidos ao implante de marca-passo, nos períodos pré, trans e pós-operatórios.

\section{Avaliação clínica do paciente no pré-operatório (14)}

Ouvir queixas álgicas no uso de sistemas de alta

12 energia no transtorácico avaliando o grau de dor do paciente. transtorácico com sistemas de alta energia frente às suas 1 queixas álgicas.

\section{Avaliação cirúrgica do paciente e de gestão do cuidado Perioperatório (29)}

7 Abrir o material que será utilizado obedecendo aos princípios estéreis.

Circular a sala, provendo materiais e equipa-

20 mentos à equipe cirúrgica conforme princípios de assepsia.

25 Assumir controles gerenciais mensurando indicadores próprios específicos para o setor de MP.
0,5 Circular a sala, provendo materiais e equipamentos à equipe cirúrgica conforme princípios de assepsia. tensão arterial sistêmica; rebaixamento de nível de consciência; oligúria; pulsos filiformes e extremidades frias.

Mensurar indicadores de qualidade e quantidade de assistência ao portador de marca-passo.

\section{Assistência no pós-operatório (20)}

5 Cuidados com drenos no local cirúrgico.

9 Avaliação sistêmica deperfusão e pulsos.

Possibilitar avaliação do conhecimento, avalia-

8 ções do estilo de vida; realizando a integração entre os pacientes e qualidade de vida.
Realizar cuidados no local de incisão cirúrgica de avaliação do enfermeiro.

Avaliar periodicamente aperfusão capilar $<3$ segundos e controle de pulsos periféricos dosmembros superiores.

Orientar sobre os cuidados no pós-operatório relacionados ao seu estilo e qualidade de vida, à possibilidade de interação com outros pacientes portadores de marca-passo, mediante avaliação do conhecimento prévio e necessidade do paciente.

\section{Identificação, intervenção frente às principais complicações (13)}

Preparar para reprogramação de novo MP após antibioticoterapia pós $72 \mathrm{~h}$ de cultura negativa, se infecção valvar e conforme indicação médica ou protocolo institucional.
Prestar assistência ao paciente com indicação de reimplante de acordo com protocolo institucional.

\section{Ação educativa ao paciente (32)}

$\begin{array}{llll}1 \text { Promover terapêutica da relação de confiança } & 0,66 & \begin{array}{l}\text { Promover a relação de confiança enfermeiropaciente no cui- } \\ \text { enfermeiro-paciente. }\end{array} \\ 8 \text { Avaliar a adesão terapêutica. } & 0,66 \quad \begin{array}{l}\text { Avaliar a adesão terapêutica medicamentosa por meio de es- } \\ \text { calas validadas e recomendadas pela Sociedade Brasileira de } \\ \text { Cardiologia. }\end{array}\end{array}$

19

Orientar e supervisionar cuidados com aparelho de MP.
Orientar e supervisionar cuidados com aparelho de marca-passo no pré, trans e pós-operatório (pele; inserção; equipamento; aspectos assépticos; parâmetros e acessórios). 


\section{Assistência aos familiares (12)}

1 Promover terapêutica da relação de confiança enfermeiro familiar.
0,57

0,57
Promover terapêutica da relação de confiança entre enfermeiro-familiar esclarecendo sobre corresponsabilidade do cuidado, dúvidas e integrando-o no processo do cuidado.
6 Proporcionar ao paciente a periodicidade e regularidade do tratamento proposto.
Orientar o paciente e familiar sobre a importância da periodicidade e regularidade no seguimento do tratamento proposto.
1

Fonte: Informações Coletadas a partir do Formulário elaborado para pesquisa. 2017.

\section{DISCUSSÃO}

Após duas rodadas de avaliação, verificou-se que o item 8 da "Competências relacionadas a conhecimentos da enfermagem para promoção de assistência segura", foi o que apresentou baixo nível de concordância devido à falta de clareza e especificidade. Considerou-se essencial o conhecimento do enfermeiro acerca dos cuidados ao paciente que será submetido à implantação de marca-passo durante os períodos pré, trans e pósoperatório, a história pregressa do paciente, os riscos que podem levar de uma arritmia precedente a uma grave complicação, nos períodos pré, trans e pos-implantação. ${ }^{4}$ Constatou-se em literatura, a relação entre a incidência de complicações com a experiência dos profissionais, considerando que profissionais com pouca experiência na área expunham o paciente a maior incidência de complicações. ${ }^{11}$

Sugeriu-se no item 12, referente à avaliação do paciente nos cuidados pré-operatório, inserir a avaliação do enfermeiro frente à dor ao que foi atendido, considerando-se que a estimulação cardíaca temporária, além da estimulação transtorácica cutânea, tem sua maior aplicação na emergência, podendo provocar dor e é utilizado enquanto se providencia o marca-passo transvenoso. ${ }^{12}$

Note-se que no item 7 , relacionado ao cuidado perioperatório, a qualidade e a segurança do paciente, é de responsabilidade de todos os profissionais, inclusive da equipe de enfermagem, que tem papel fundamental na prevenção da ocorrência de eventos adversos. ${ }^{13}$ Sabe-se, dessa forma, a importância em conhecer os materiais específicos, realizar os testes dos equipamentos antes do procedimento e seguir a técnica asséptica, a fim de prevenir complicações. ${ }^{4,14}$ Verificou-se em um estudo com implantação de 160 marcapassos, que $10 \%$ dos pacientes submetidos ao procedimento apresentou infecção no local após 75 dias da implantação do dispositivo, o que pode ser minimizado por medidas de antissepsia e cuidados pós-operatórios. ${ }^{14}$

Quanto ao item 20, recomendou-se especificar uma anamnese direcionada a fim de atender às necessidades e executar uma prescrição adequada na evolução do paciente, o que coaduna com a literatura. ${ }^{1}$

Modificou-se ainda o item 25, ao considerar-se que as complicações mais comuns para implantação de marca-passo são punção arterial, hematoma, infecção da loja do gerador, deslocamento do eletrodo e pneumotórax15 e, ao relacionar indicadores, que são medidas utilizadas para descrever uma situação, avaliar mudanças durante um período de tempo e ações realizadas, ${ }^{16}$ relevantes para mensuração da qualidade do cuidado e proposição de intervenções.

Notou-se ainda, a necessidade de alteração dos itens 5, 9 e 20 das competências relacionadas aos cuidados no pós-operatório, e do item 8 das competências relacionadas às principais complicações. Observou-se que alguns cuidados no pós-operatório, que são importantes ao conside- rar que uma punção venosa, podem apresentar complicações como pneumotórax ou hemotórax, além de se atentar ao posicionamento dos eletrodos, com realização de radiografia de tórax, ECG e visão clínica do enfermeiro sob o paciente. ${ }^{17}$ Julgou-se que o tempo de internação geralmente é curto, sendo relevante exercer o autocuidado do paciente. ${ }^{18}$

Em relação aos itens 1, 8 e 19 das competências relacionadas à ação educativa ao paciente e aos familiares, indicou-se melhorar a especificação de cada item devido à importância do papel do enfermeiro como orientador em todas as etapas, pré, trans e pósimplante, além de todos os procedimentos para o auto-cuidado. ${ }^{18}$ Mostrou-se em um artigo publicado em 2012, que 56 pacientes demonstraram pouco conhecimento sobre as implicações em caso de falha do Dispositivo Cardíaco Eletrônico Implantável (DCEI). ${ }^{19}$ Apontou-se em um estudo feito em 2015, uma amostra de 114 pacientes idosos que apresentaram depressão e ansiedade relativamente graves antes e após a implantação do marca-passo, e após o atendimento contínuo, os níveis de depressão e ansiedade diminuíram significativamente, ${ }^{20}$ o que justificou a necessidade de avaliar o conhecimento do paciente e prática de seu auto-cuidado.

\section{CONCLUSÃO}

As competências profissionais do enfermeiro nos cuidados com portadores de marcapasso e seus itens de cuidado foram validadas quanto à 
confiabilidade de seu conteúdo, com duas rodadas de avaliação junto aos juízes.

Considerou-se que a validação destas competências profissionais por juízes pode contribuir para a prática diferenciada, realizando o cuidado com qualidade, para prevenir danos, promover a saúde, além de fortalecer as competências na cardiologia, contemplando cuidados na prevenção, tratamento e promoção de educação ao usuário e família.

Torna-se necessária a continuidade do estudo com a validação clínica destas competências no cotidiano do enfermeiro atuante na área assistencial ao portador de marcapasso.

Sabe-se que o exercício profissional nessa área é uma prática rotineira que exige da equipe de enfermagem domínio e visão clínica para atender esses portadores, sendo fundamental para prevenir danos, e se fazem necessárias mais publicações quanto às competências profissionais a fim de embasar cientificamente as práticas clínicas.

*Caso tenha interesse em ver a Lista modificada de competências profissionais do enfermeiro nos cuidados com portadores de marca-passo, envie um e-mail para sergioh@idpc.org.br

\section{Referências}

1. World Health Organization. A global brief on hypertesion. Silent killer, global public health crisis. [Internet]. Geneva: WHO; 2013 [cited 2018 Oct 30]. Available from http://apps.who.int/iris/bitstre$\mathrm{am} / \mathrm{handle} / 10665 / 79059 / \mathrm{W}$ ? sequence $=1$

2. World Health Organization. Hearts: technical package for cardiovascular disease management in primary health care. [Internet]. Geneva: WHO; 2016 [cited 2018 Apr 29]. Available from: http://apps. who.int/iris/bitstream/handle/10665/252661/9789241511377eng.pdf?sequence

3. Sociedade Brasileira de Cirurgia Cardiovascular. Diretrizes Brasileiras de Dispositivos Cardíacos Eletrônicos Implantáveis do Departamento de Estimulação Cardíaca Artificial (DECA) da Sociedade Brasileira de Cirurgia Cardiovascular (SBCCV). Departamento de Estimulação Cardíaca Artificial da Sociedade Brasileira de Cirurgia Cardiovascular (DECA/SBCCV). [Internet]. 2015 [cited 2016 Sep 10]. Available from: http://www.deca.org.br/Medica/arquivos/ SuplementoRelampa.pdf

4. Mulpuru SK, Madhavan M, McLeod CJ, Cha YM, Friedman PA. Cardiac Pacemakers: Function, Troubleshooting, and Management. J Am Coll Cardiol. 2017;69(2):189-210. DOI: 10.1016/j. jacc.2016.10.061

5. Santos FCP, Rubio EN, Abreu LF, Barouche ER, Mateos JCP, Mateos EIP, et al. Marcapasso cardíaco: quando indicar e como usar. Rev Fac Ciênc Méd Sorocaba [Internet]. 2008 [cited 2016 Sep 10];10(4):5-7. Available from: http://revistas.pucsp.br/index.php/ RFCMS/article/viewFile/698/985

6. Raatikainen MJP, Arnar DO, Zeppenfeld K, Merino JL, Levya F, Hindriks $G$, et al. Statistics on the use of cardiac electronic devices and electrophysiological procedures in the European Society of Cardiology countries: 2014 report from the European Heart Rhythm Association. Europace. 2015;17:i1-i75. DOI:10.1093/europace/euu300

7. Kobayashi RM. A construção de competências profissionais de enfermeiros em serviço num hospital de ensino [Tese]. São Paulo: Escola de Enfermagem, Universidade de São Paulo; 2007.

8. Steffes SS, Thompson EA, Bridges EM, Dougherty CM. Knowledge of Implantable Cardioverter Defibrillator Purpose and Function Among Nurses in the United States. Journal of Cardiovascular Nursing. J Cardiovasc Nurs. 2017 May/Jun;32(3):304-10. DOI: 10.1097/ JCN.0000000000000339

9. Kobayashi RM, Simonetti SH, Pascon DM. Competências Profissionais dos Enfermeiros no Cuidado ao Portador de Marca-passo. In: Competências Clínicas para Enfermeiros. São Paulo: Atheneu; 2017.
10. Polit DF, Beck CT, Owen SV. Is the CVI an acceptable indicator of content validity? Appraisal and recommendations. Res Nurs Health. 2007;30(4):459-67. DOI: 10.1002/nur.20199

11. Díaz-Miguel O, Gómez Grande ML. Temporary internal pacing. Med Intensiva. 2014;38(9):531-94. DOI: 10.1016/j.medine.2014.02.001

12. Wilkoff BL, Fauchier L, Stiles MK, Morillo CA, Al-Khatib SM, Almendral J, et al. Erratum to '2015 HRS/EHRA/APHRS/SOLAECE expert consensus statement on optimal implantable cardioverter-defibrillator programming and testing'. J Arrhythm. 2016;32(5):4412. DOI: 10.1016/j.joa.2016.08.001

13. Bohomol E, Tartali J.A. Eventos adversos em pacientes cirúrgicos: conhecimento dos profissionais de enfermagem. Acta Paul Enferm. 2013;26(4):376-81. DOI: 10.1590/S0103- 21002013000400012.

14. Gill JS, Singh N, Khanna SP. Risk of cardiac pacemaker pocket infection in a tertiary care hospital. Indian J Pathol Microbiol. 2017 Apr-Jun;60(2):185-8. DOI: 10.4103/IJPM.IJPM_190_16

15. Kirkfeldt RE, Johansen JB, Nohr EA, Jørgensen OD, Nielsen JC Complications after cardiac implantable electronic device implantations: an analysis of a complete, nationwide cohort in Denmark. Eur Heart J. 2013;35(18):1186-94. DOI: 10.1093/eurheartj/eht511

16. Rossaneis MA, Gabriel CS, Haddad MCFL, Melo MRAC, Bernardes $A$. Indicadores de qualidade utilizados nos serviços de enfermagem de hospitais de ensino. Rev Eletr Enf. 2014 out/ dez;16(4):769-76. DOI: 10.5216/ree.v16i4.22956

17. Bronchtein S. Implante de marcapasso cardíaco definitivo. Aspectos cirúrgicos. Revista da SOCERJ [Internet]. 2002 [cited 2017 Dec 7]; 15(2):102-12. Available from: http://sociedades.cardiol.br/ socerj/revista/2002_02/a2002_v15_n02_art07.pdf

18. Fernandes IR, Gallardo ALA, Zaramella VM. Marcapasso e desfibrilador implantável: avaliação do conhecimento do portador para o auto-cuidado. Arq Med Hosp Fac Cienc Med Santa Casa São Paulo [Internet]. 2015 [cited 2017 Dec 7];60(1):12-5. Available from: http://arquivosmedicos.fcmsantacasasp.edu.br/index.php/ AMSCSP/article/view/160/169

19. Braun S, Krüger J, Souza EN, Rabelo ER. Quality of life of patients with implanted cardiac devices A transversal study. OBJN. 2012;11(3):778-88. DOI: 10.5935/1676-4285.20120051

20. Liu X, He X, Li L, Haung L, Liu Z. Influence of Continuous Nursing on the Psychological State and Coping Style of Patients Undergoing Pacemaker Implantation. Iran J Public Health [Internet]. 2015 [cited 2017 Dec 10];44(7):953-61. Available from: https:// www.ncbi.nlm.nih.gov/pmc/articles/PMC4645766/ 